\title{
On the Accelerated Settling of Fine Particles in a Bidisperse Slurry
}

\author{
Leonid L. Minkov, ${ }^{1}$ Yuliya O. Stepanova, ${ }^{1}$ Johann Dueck, ${ }^{2,3}$ and Elizaveta V. Pikushchak ${ }^{1}$ \\ ${ }^{1}$ Tomsk State University, 36 Lenin Avenue, Tomsk 634050, Russia \\ ${ }^{2}$ University of Erlangen-Nuremberg, 3 Paul-Gordan-Strasse, 91052 Erlangen, Germany \\ ${ }^{3}$ Department of Natural Sciences, L.N. Gumilyov Eurasian National University, Munaitpasov Street 5, Astana 010008, Kazakhstan
}

Correspondence should be addressed to Leonid L. Minkov; lminkov@ftf.tsu.ru

Received 5 November 2014; Accepted 15 March 2015

Academic Editor: George S. Dulikravich

Copyright (C) 2015 Leonid L. Minkov et al. This is an open access article distributed under the Creative Commons Attribution License, which permits unrestricted use, distribution, and reproduction in any medium, provided the original work is properly cited.

\begin{abstract}
An estimation of increasing the volume average sedimentation velocity of fine particles in bidisperse suspension due to their capturing in the circulation zone formed in the laminar flow of incompressible viscous fluid around the spherical coarse particle is proposed. The estimation is important for an explanation of the nonmonotonic shape of the separation curve observed for hydrocyclones. The volume average sedimentation velocity is evaluated on the basis of a cellular model. The characteristic dimensions of the circulation zone are obtained on the basis of a numerical solution of Navier-Stokes equations. Furthermore, these calculations are used for modelling the fast sedimentation of fine particles during their cosedimentation in bidisperse suspension. It was found that the acceleration of sedimentation of fine particles is determined by the concentration of coarse particles in bidisperse suspension, and the sedimentation velocity of fine fraction is proportional to the square of the coarse and fine particle diameter ratio. The limitations of the proposed model are ascertained.
\end{abstract}

\section{Introduction}

In a number of industries (mining, chemical, food, etc.) machines based on the principle of settling (sedimentation) of particles in a rotating fluid flow are used for the separation of particulate solids from air or liquid (air cyclones, hydrocyclones, centrifuges, decanters, etc.) [1].

Mathematical models for processes in such devices have long been developed that achieve impressive results. For example, Reynolds stress model [2-4] and Large Eddy Simulation approach [5-7] are employed to describe the turbulent flow field in cyclones. Particularities of solid phase motion are specified with discrete element model $[2,8]$, discrete phase model $[4,9]$, mixture model $[10,11]$, or Euler model $[12,13]$. Even so, a number of effects are in principle incapable of being clearly mathematically described. Such effects include abnormal behavior of the so-called separation efficiency function, that is, the portion of the separated fraction of initial material depending on the particle size of the fraction.
This phenomenon is often a hindrance for engineers using hydrocyclones because the sharpness of fractionation often deteriorates due to the fine particles (in practice, particles smaller than 10 microns) contrary to expectations falling into the coarse product. Anomalous behavior of the separation function is particularly noticeable for the operation of small hydrocyclones, where the quality of the fine particle separation efficiency deteriorates, leading to an unexpected increase in the separation function with decreasing particle size ("fishhook" effect) [1].

Abnormal growth of the separation function is the subject of lively debate. Various theories to explain this effect have been developed by now. One of them is based on a variable bypass to underflow of hydrocyclone [14, 15]; another is "mechanistic" model based on estimation of particle settling velocity depending on the number of Re [16]. Fluid mechanics based models, namely, the boundary layer model [17] and entrainment models [10, 18], which assign anomalous behaviour of the separate function to a hydrodynamic 
interaction of fine and coarse particles should be noted. A comprehensive critical analysis of existing models explaining "fish-hook" phenomenon has recently been published [19], where opposed to [20] the effect is attributed to measurement errors.

The most likely reason for this anomaly, in our opinion, is connected with the fact that there is an acceleration of sedimentation of fine particles under the influence of neighboring coarse particles in the polydisperse suspension. Due to an entrainment the fine particles have a much higher sedimentation velocity than expected and are intensively removed together with a coarse fraction from the hydrocyclone [18]. The mechanism of the accelerated sedimentation of fine particles has not been studied well enough.

To explain this effect, a model of the cell for bidisperse suspension has been proposed [21]. That model relies on the determination of the mean residence time of small particles in the cell surrounding the large ones and the subsequent determination of an average sedimentation velocity of small particles. Accelerated sedimentation of small particles occurs due to their retention in the flow boundary layer near the surface of the large particle. Herein the flow around the large particle is postulated identical to the Stokes flow that is valid for $U_{c} d_{c} / \nu_{l}<1$. An analysis of the effect of the Reynolds number on the sedimentation velocity of the particles and consequently on the hydrocyclone separation curve has been carried out [22], but it has not considered the particleparticle interaction phenomena which is so important in the range of the finest particles. Further development of the sedimentation model followed the path of propagation of the entrainment mechanism of small particles by the large ones for the case of polydisperse suspension, using it to evaluate the separation function of the hydrocyclone.

Thus, it was possible to calculate the separation function, including its nonmonotonic character [23]. At the same time it becomes clear that some large particles in suspension in a hydrocyclone can move at Re of the order of $10^{2}$. It is known [24] that the boundary layer around the moving particles in a liquid is radically changed already at $\operatorname{Re} \approx$ 25: instead of the smooth streamlines along the direction of particles motion a zone of reverse flows appears. They become more complicated for the increased Reynolds number. This requires further development of the entrainment model.

The logical explanation for the accelerated sedimentation of small particles in the presence of large ones can be considered as a capture of small particles entering the hydrodynamic wake generated by a large particle at the numbers of $\operatorname{Re}>25$.

The aim of this paper is to study the circulation zone that occurs in the laminar flow of an incompressible viscous fluid around a spherical particle and the determination of the volume average sedimentation velocity of small particles during the sedimentation of bidisperse suspension, based on a cellular model.

\section{Mathematical Formulation of the Problem}

The system of Navier-Stokes equations describing the steady state laminar flow of an axisymmetric incompressible viscous

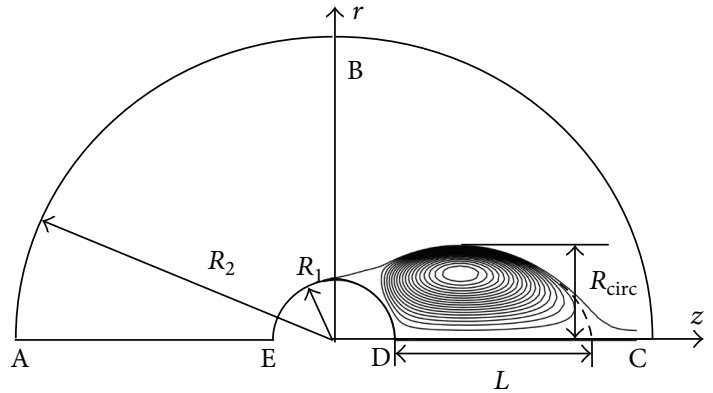

FIGURE 1: Domain of integration.

fluid around a sphere in a cylindrical coordinate system is as follows [25]:

$$
\begin{gathered}
V_{r} \frac{\partial V_{r}}{\partial r}+V_{z} \frac{\partial V_{r}}{\partial z}+\frac{1}{\rho} \frac{\partial p}{\partial r}=\nu\left(\nabla^{2} V_{r}-\frac{V_{r}}{r^{2}}\right), \\
V_{r} \frac{\partial V_{z}}{\partial r}+V_{z} \frac{\partial V_{z}}{\partial z}+\frac{1}{\rho} \frac{\partial p}{\partial z}=\nu\left(\nabla^{2} V_{z}\right), \\
\frac{1}{r} \frac{\partial}{\partial r}\left(r V_{r}\right)+\frac{\partial V_{z}}{\partial z}=0
\end{gathered}
$$

where

$$
\nabla^{2} \equiv \frac{1}{r} \frac{\partial}{\partial r}\left(r \frac{\partial}{\partial r}\right)+\frac{\partial^{2}}{\partial z^{2}} .
$$

The domain of integration of the system of (1), presented in Figure 1, is limited by the input boundary AB, the output boundary $\mathrm{BC}$, and the axis of symmetry DC $\cup \mathrm{AE}$, as well as the contour of the sphere ED.

The boundary conditions are specified as follows.

The solid wall (ED) is

$$
\begin{aligned}
-R_{1}<z<R_{1}, & r=\sqrt{R_{1}^{2}-z^{2}}, \\
V_{r}=0, & V_{z}=0 .
\end{aligned}
$$

The input boundary $(\mathrm{AB})$ is

$$
\begin{aligned}
-R_{2}<z<0, & r=\sqrt{R_{2}^{2}-z^{2}}, \\
V_{r}=0, & V_{z}=U_{0} .
\end{aligned}
$$

The output boundary (BC) is

$$
\begin{gathered}
0<z<R_{2}, \quad r=\sqrt{R_{2}^{2}-z^{2}}, \\
\frac{\partial V_{r}}{\partial z}=0, \quad \frac{\partial V_{z}}{\partial z}=0 .
\end{gathered}
$$

The axis of symmetry AE $\cup \mathrm{DC}$ is

$$
\begin{aligned}
& -R_{2}<z<-R_{1}, \quad R_{1}<z<R_{2}, \\
& V_{r}=0, \quad \frac{\partial V_{z}}{\partial r}=0, \quad \frac{\partial p}{\partial r}=0 .
\end{aligned}
$$




\section{Method of Solving the Problem}

A solution of the system (1) was carried out using Patankar finite difference scheme [26] with the SIMPLE procedure to couple the pressure and velocity fields of the fluid and the second order upwind scheme to determine the convective flows.

The implementation of this method was carried out using the software package ANSYS-Fluent. For the construction of the finite difference grid, the program Gambit was used.

The ratio of the radii of boundaries ABC and ED is set as a constant, $R_{2} / R_{1}=14$. Preliminary calculations show that for larger values of $R_{2} / R_{1}$ no changes in the calculation results take place.

Special research found that sufficient accuracy of calculations (with an error of $2 \%$ ) at $\mathrm{Re}=100$ is achieved by using a computational grid of 40,000 cells.

In particular, the experimental Schiller-Naumann correlation [27] for the drag coefficient of a sphere $C_{D}=$ $(24 / \mathrm{Re}) f_{D}(\mathrm{Re})$, where $f_{D}(\mathrm{Re})=1+0.15 \mathrm{Re}^{0.687}$, is fully reproduced by the numerical solution.

The difference in the results of the numerical solutions and the experimental data does not exceed $5 \%$ for $\mathrm{Re} \leq 200$. At $\operatorname{Re}=1000$ the calculated value of $C_{D}$ is lower than the experimental one by $23.7 \%$. The deviation of the calculated drag coefficient from the experimental one can be explained by the fact that at high values of the Re number (about 1000) theturbulization of the flow begins to manifest itself, which is not taken into consideration in the calculations.

\section{Dependence of the Circulation Zone Size on the Reynolds Number}

As was mentioned above when the Reynolds number is greater than 25 a circulation zone is formed at the rear of the sphere $[24,28,29]$ which is caused by the detachment of the boundary layer from the surface of the sphere (Figure 1). This fact is confirmed by our calculations.

Below we limit ourselves to describing only those geometric characteristics of the circulation zone, which are needed for the later described model of the particle mixture sedimentation.

The dependence of the volume of the circulation zone related to the volume of the sphere on the Reynolds number is presented in Figure 2. The relative volume of the circulation zone is approximated by a polynomial of the second degree in the range of $\mathrm{Re}=25-1000$ with a correlation coefficient of $R^{2}=0.9998$

$$
W(\mathrm{Re})=-0.258+1.017 \frac{\mathrm{Re}}{100}-3.78 \cdot 10^{-2}\left(\frac{\mathrm{Re}}{100}\right)^{2}
$$

or by an exponential function with a correlation coefficient of $R^{2}=0.9993$ :

$$
W(\mathrm{Re})=9.194 \cdot \exp \left[-0.777 t^{3}+2.963 t^{2}-4.723 t\right],
$$

where $t=100 /$ Re.

As follows from (7), (8), and Figure 2, the circulation zone begins to form at $\mathrm{Re}_{*}=25.6$.

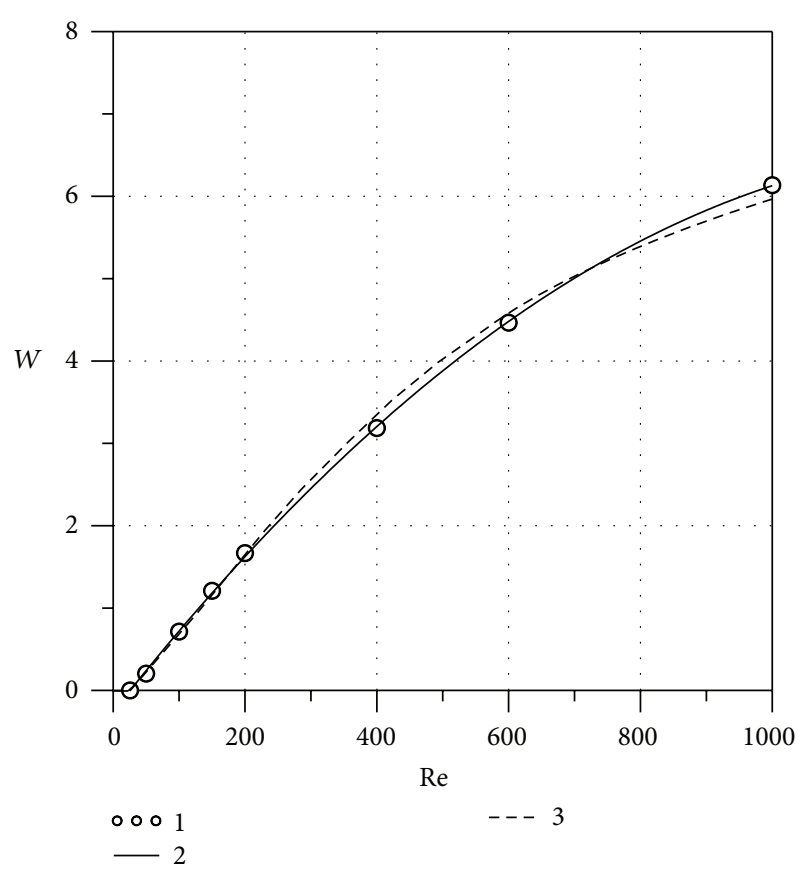

FIGURE 2: Dependence of the circulation zone volume on the Reynolds number. 1: numerical experiment, 2: approximation curve, (7), and 3: approximation curve, (8).

The size of the circulation zone can be characterized by its length $L$ (related to the diameter of the sphere) defined as the distance from the rear point of the sphere $D$ to the intersection point of the limit streamline of circulation zone and the axis of symmetry (Figure 1).

Figure 3 shows the dependence of the length of the circulation zone $L$ on the Reynolds number. The results of the numerical simulation shown as circles are approximated by an exponential with a correlation coefficient of $R^{2}=0.9997$ :

$$
L(\mathrm{Re})=2.858 \cdot \exp \left(-\frac{28.778}{\mathrm{Re}^{0.7}}\right)
$$

It is similarly possible to establish the dependence of another important parameter, namely, the radius of circulation zone (Figure 1), on the Reynolds number, which is approximated by the formula $R_{\text {circ }} / R_{1}=0.218 \mathrm{Re}^{0.291}$ in the range of $\mathrm{Re}=50-1000$ with a correlation coefficient of $R^{2}=$ 0.9985 .

The results of experiments obtained by Taneda [29] in the range of Reynolds number from 40 to 100 are depicted in Figure 3 as triangles. A good correlation between the calculated and experimental data is observed. With an increase in the Reynolds number from 10 to 1000, an increase in length of the circulation zone is observed. The rate of growth $L(\mathrm{Re})$ reduces when the values of Re are approaching 1000.

Thus, the relative length and the relative volume as functions of the Reynolds number can characterize the circulation zone at the rear of the sphere. 


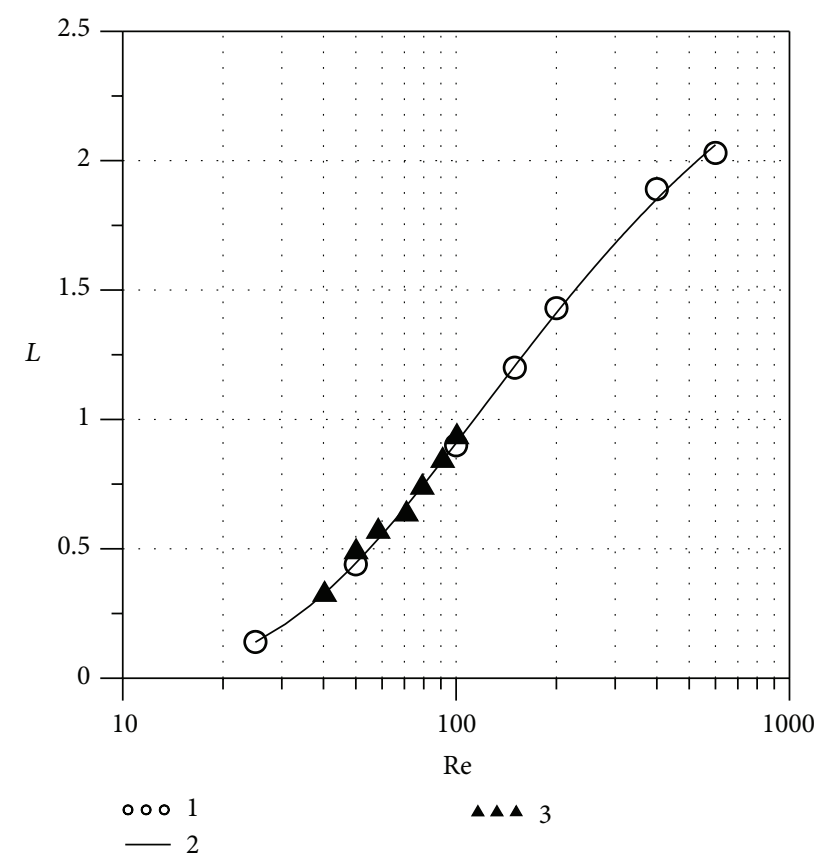

FIGURE 3: Dependence of the circulation zone length on the Reynolds number. 1: numerical experiment, 2: approximation curve, and 3: experimental data [29].

\section{Sedimentation Velocity of Fine Particles in Bidisperse Suspension}

The sedimentation of a bidisperse suspension (a mixture of coarse particles with diameter $d_{c}$ and fine particles with diameter $d_{f}$ ) is considered. Around each coarse particle of radius $R_{1}$, we construct a spherical cell of radius $R_{2}$ (Figure 1 ).

We suppose that, during the cosettling of coarse and fine particles, the latter trapped in the circulation zone formed at the rear of the coarse particle have the same sedimentation velocity as the coarse $U_{c}$, and other fine particles settle at their own sedimentation velocity $U_{f}$.

Let us assume that the considered cell has a volume $v_{\text {cell }}$ and the circulation zone has the volume $v_{\text {cyrc }}<v_{\text {cell }}$. Then the volume average sedimentation velocity of fine particles in the cell is determined by the following expression:

$$
\left\langle U_{f}\right\rangle=\frac{v_{\mathrm{sph}} W(\mathrm{Re})}{v_{\text {cell }}-v_{\mathrm{sph}}} U_{c}+\frac{v_{\text {cell }}-v_{\mathrm{sph}} W(\mathrm{Re})}{v_{\text {cell }}-v_{\mathrm{sph}}} U_{f},
$$

where $v_{\mathrm{sph}}$ is the volume of the coarse particle and $\mathrm{Re}=$ $d_{c} U_{c} / \nu$.

The equilibrium equation of forces acting on a particle during the sedimentation allows one to obtain its velocity:

$$
U=\frac{\rho_{s}-\rho_{l}}{\rho_{l}} \frac{g \cdot d^{2}}{18 \nu} \frac{1}{f_{D}(\mathrm{Re})} .
$$

For fine particles which can be considered as the Stokes ones $f_{D}(\mathrm{Re})=1$ and $U_{f} \sim d_{f}^{2}$. For coarse particles $U_{c} \sim$ $d_{c}^{2} / f_{D}(\mathrm{Re})$.
Therefore,

$$
\frac{\left\langle U_{f}\right\rangle}{U_{f}}=\frac{v_{\mathrm{sph}}}{v_{\text {cell }}-v_{\mathrm{sph}}} \frac{W(\mathrm{Re})}{f_{D}(\mathrm{Re})}\left(\frac{d_{c}}{d_{f}}\right)^{2}+\frac{v_{\text {cell }}-v_{\mathrm{sph}} W(\mathrm{Re})}{v_{\text {cell }}-v_{\mathrm{sph}}} .
$$

As a volume fraction of the coarse particles in the suspension is expressed through the volume of the cell and the own volume of coarse particle by the relation $\alpha_{\mathrm{c}}=$ $v_{\text {sph }} / v_{\text {cell }}$, then

$$
\frac{\left\langle U_{f}\right\rangle}{U_{f}}=\frac{1}{1-\alpha_{c}}+\frac{\alpha_{c} W(\mathrm{Re})}{1-\alpha_{c}}\left(\frac{1}{f_{D}(\mathrm{Re})}\left(\frac{d_{c}}{d_{f}}\right)^{2}-1\right) .
$$

As can be seen from (13), the volume average sedimentation velocity of fine particles increases proportionally to the square of the coarse and fine particle diameter ratio which correlates with the experimental data obtained for the settling of water-sand suspension in a plate centrifuge [30] and for the droplet creaming in a tube [31].

From (7), (8), and (13) it also follows that the volume average sedimentation velocity of fine particles increases monotonically with increasing the volume fraction of the coarse particles. Moreover, the acceleration of sedimentation of fine particles at small $\alpha_{c}$ is proportional to $\alpha_{c}$, in contrast to the flow at a low $\mathrm{Re}$, where the acceleration is proportional to $\alpha_{c}^{1 / 3}$ [21].

An analysis of (13) shows that for fixed $d_{c} / d_{f}$ the dependence of the volume average sedimentation velocity of fine particles on the Reynolds number is nonmonotonic. With the increasing of the Reynolds number the velocity increases, reaching a maximum, and then decreases. Note that changing the Reynolds number in real devices is accomplished by setting the level of the tangential velocity through the inlet pressure variation.

The limits of the applicability of formula (9) are determined by the fact that the circulation zone should be completely inside the spherical cell; that is, $d_{c} L(\mathrm{Re})+d_{c} / 2<$ $R_{2}$, whence it follows that a formal limitation on the volume fraction of coarse particles depends on the Reynolds number:

$$
\alpha_{c} \leq \frac{1}{[1+2 L(\mathrm{Re})]^{3}} .
$$

As follows from (13) the maximum growth of the volume average sedimentation velocity of fine particles during the settling together with coarse particles is obtained at the maximum possible value of $\alpha_{c}$, which is determined by (14) $\alpha_{m}(\mathrm{Re})=[1+2 L(\mathrm{Re})]^{-3}$, Figure 4.

In the region of practical interest, $\mathrm{Re}$ is about $10^{2}$ and $\alpha_{m}$ may be around a few percent. This is the typical range of the coarse and fine particles concentration ratio in the suspension. 


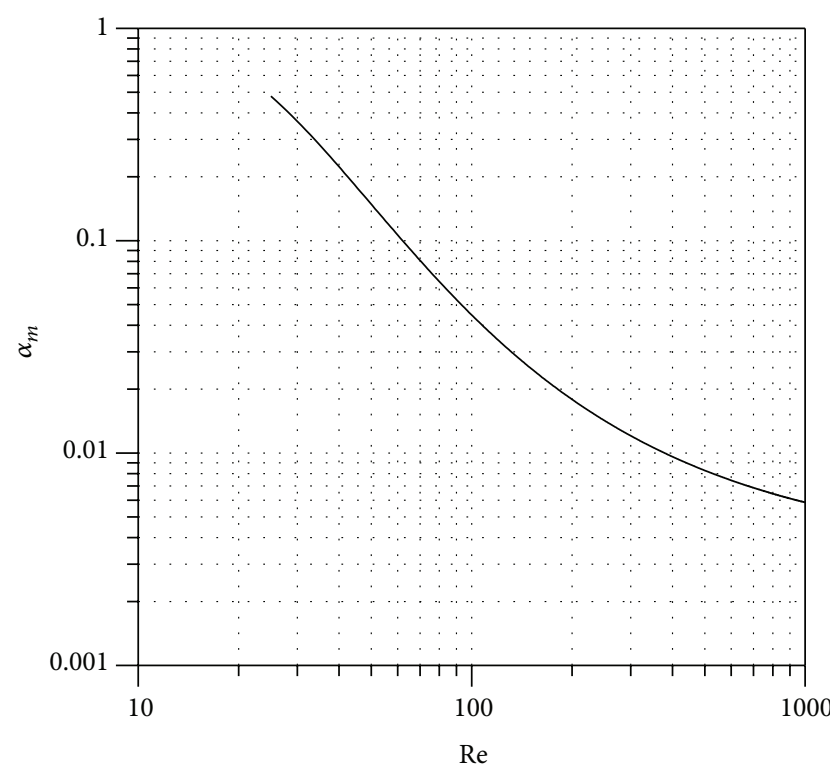

FIgURE 4: Maximum possible volume fraction of coarse particles.

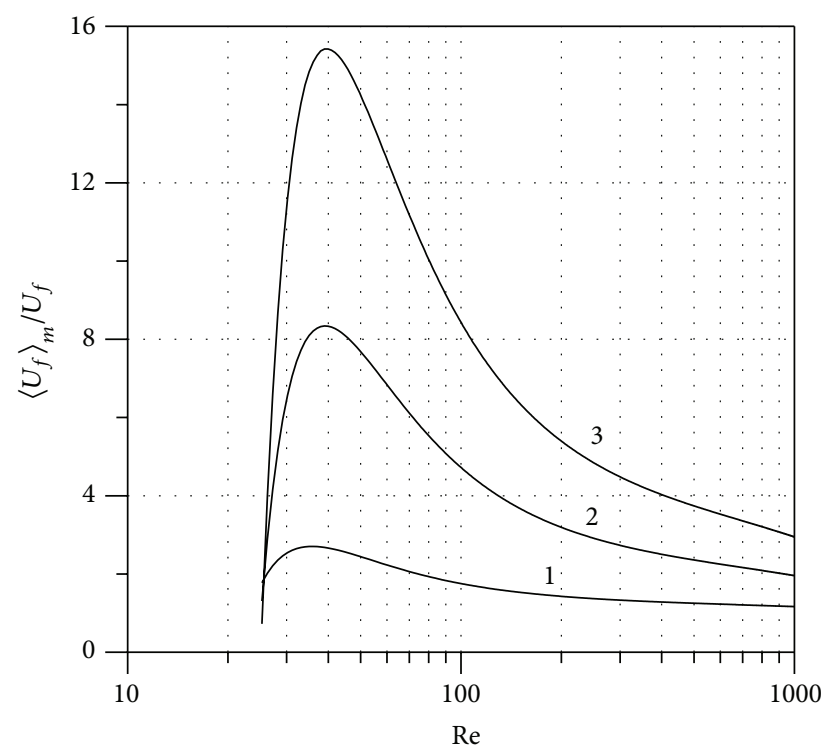

FIGURE 5: Maximum possible volume average sedimentation velocity of fine particles. $1-\left(d_{c} / d_{f}\right)^{2}=100,2-500,3-1000$.

Equations (13) and (14) give a maximum of the average sedimentation velocity of fine particles depending on Re:

$$
\begin{aligned}
\frac{\left\langle U_{f}\right\rangle_{m}}{U_{f}}= & \frac{1}{1-\alpha_{m}(\mathrm{Re})} \\
& +\frac{\alpha_{m}(\mathrm{Re}) W(\mathrm{Re})}{1-\alpha_{m}(\mathrm{Re})}\left(\frac{1}{f_{D}(\mathrm{Re})}\left(\frac{d_{c}}{d_{f}}\right)^{2}-1\right) .
\end{aligned}
$$

Figure 5 shows the dependence of the maximum value of the volume average sedimentation velocity of fine particles on the Reynolds number for different values of $\left(d_{c} / d_{f}\right)^{2}$. It is evident that the dependence is nonmonotonic due to the different rates of increasing the length and volume of the circulation zone with an increasing Reynolds number.

It is noteworthy that the increase in the sedimentation velocity of fine particles by described mechanism is limited and should not be expected to be higher than 10 times.

Equation (9) taken at $\mathrm{Re}=\mathrm{Re}_{*}$ allows one to determine the minimum size $d_{c \text {, min }}$ of coarse particle behind which a circulation zone can be formed:

$$
d_{c, \text { min }}=\left(\operatorname{Re}_{*} \cdot f_{D}\left(\operatorname{Re}_{*}\right) \frac{\rho_{l}}{\left(\rho_{s}-\rho_{l}\right)} \frac{18 v^{2}}{g}\right)^{1 / 3}
$$

The circulation zone under operating conditions of a hydrocyclone (let us take $g=1000 \mathrm{~m} / \mathrm{s}^{2}$ ) with a quartz water-sand suspension is formed for particles larger than 88 microns and in the gravitational settling for particles larger than 400 microns. Thus, the described mechanism of accelerating the particles sedimentation in a suspension can probably be realized if the suspension includes particularly large particles.

\section{Conclusion}

Based on a numerical simulation of the laminar flow of incompressible viscous fluid around a spherical particle the geometric characteristics (volume and length) of the circulation zone formed in the rear of the sphere depending on the Reynolds number for $25<\operatorname{Re}<1000$ were obtained.

The formula for the volume average sedimentation velocity of fine particles settling in the presence of coarse particles (in the case of bidisperse suspension) under the assumption that the fine particles trapped in the circulation zone settle at a velocity of coarse ones was obtained. An expression for the maximum sedimentation velocity of fine particles shows nonmonotonic behavior depending on the Reynolds number.

\section{Nomenclature}

d: Particle diameter

$f_{D}$ : Drag function

g: Acceleration

p: Pressure

$t$ : Time

$r, z$ : Spatial coordinates

$C_{D}$ : Drag coefficient

$L: \quad$ Relative length of circulation zone

$R: \quad$ Radius

Re: Reynolds number

$\mathrm{Re}_{*}$ : Reynolds number at which the recirculation zone appears (equal to 25)

$U: \quad$ Particle velocity

$V: \quad$ Fluid flow velocity

$W$ : Relative volume of circulation zone

$\alpha$ : Volume fraction

$\nu$ : Kinematic viscosity

$\rho:$ Density

$v$ : Volume. 


\section{Subscripts}

\section{1: $\quad$ Boundary ABC \\ 2: Boundary ED \\ c: $\quad$ Coarse particle}

cell: Cell

$f: \quad$ Fine particle

$l$ : $\quad$ Liquid

$m$ : Maximum

min: Minimum

$r: \quad$ Radial direction

$s: \quad$ Solid

sph: Sphere

$z$ : Axial direction.

\section{Conflict of Interests}

The authors declare that there is no conflict of interests regarding the publication of this paper.

\section{Acknowledgments}

Support for this work was provided by the Ministry of Education and Science of Russian Federation (agreement no. 10.1329.2014/K) and Tomsk State University Competitiveness Improvement Program.

\section{References}

[1] K. Legenhausen, Particle Classification, Chapman \& Hall, London, UK, 1993.

[2] A. J. Hoekstra, J. J. Derksen, and H. E. A. Van Den Akker, "An experimental and numerical study of turbulent swirling flow in gas cyclones," Chemical Engineering Science, vol. 54, no. 13-14, pp. 2055-2065, 1999.

[3] K. W. Chu, B. Wang, A. B. Yu, A. Vince, G. D. Barnett, and P. J. Barnett, "CFD-DEM study of the effect of particle density distribution on the multiphase flow and performance of dense medium cyclone," Minerals Engineering, vol. 22, no. 11, pp. 893909, 2009.

[4] O. S. Motsamai, "Investigation of the influence of hydrocyclone geometric and flow parameters on its performance using CFD," Advances in Mechanical Engineering, vol. 2010, Article ID 593689, 12 pages, 2010.

[5] M. Narasimha, M. Brennan, and P. N. Holtham, "Large eddy simulation of hydrocyclone-prediction of air-core diameter and shape," International Journal of Mineral Processing, vol. 80, no. 1, pp. 1-14, 2006.

[6] M. Saidi, R. Maddahian, B. Farhanieh, and H. Afshin, "Modeling of flow field and separation efficiency of a deoiling hydrocyclone using large eddy simulation," International Journal of Mineral Processing, vol. 112-113, pp. 84-93, 2012.

[7] M. D. Slack, R. O. Prasad, A. Bakker, and F. Boysan, "Advances in cyclone modelling using unstructured grids," Chemical Engineering Research and Design, vol. 78, no. 8, pp. 1098-1104, 2000.

[8] K. W. Chu, S. B. Kuang, A. B. Yu, and A. Vince, "Particle scale modelling of the multiphase flow in a dense medium cyclone: effect of fluctuation of solids flowrate," Minerals Engineering, vol. 33, pp. 34-45, 2012.
[9] F. J. de Souza, R. de Vasconcelos Salvo, and D. A. de Moro Martins, "Large Eddy Simulation of the gas-particle flow in cyclone separators," Separation and Purification Technology, vol. 94, pp. 61-70, 2012.

[10] L. Minkov, J. Dueck, and T. Neesse, "Computer simulations of the Fish-Hook effect in hydrocyclone separation," Minerals Engineering, vol. 62, pp. 19-24, 2014.

[11] J. G. Dueck, O. V. Matvienko, and T. Neesse, "Modeling of hydrodynamics and separation in a hydrocyclone," Theoretical Foundations of Chemical Engineering, vol. 34, no. 5, pp. 428-438, 2000.

[12] X. Zhang, Y. Cheng, S. Nie, H. Ji, and L. Liu, "Simulation of multiphase flow of the oil-water separation in a rotating packed bed for oil purification," Mathematical Problems in Engineering, vol. 2013, Article ID 404327, 9 pages, 2013.

[13] S. Swain and S. Mohanty, "A 3-dimensional Eulerian-Eulerian CFD simulation of a hydrocyclone," Applied Mathematical Modelling, vol. 37, no. 5, pp. 2921-2932, 2013.

[14] W. Kraipech, W. Chen, F. J. Parma, and T. Dyakowski, "Modelling the fish-hook effect of the flow within hydrocyclones," International Journal of Mineral Processing, vol. 66, no. 1-4, pp. 49-65, 2002.

[15] E. J. Roldán-Villasana, R. A. Williams, and T. Dyakowski, "The origin of the fish-hook effect in hydrocyclone separators," Powder Technology, vol. 77, no. 3, pp. 243-250, 1993.

[16] A. K. Majumder, P. Yerriswamy, and J. P. Barnwal, "The 'fishhook' phenomenon in centrifugal separation of fine particles," Minerals Engineering, vol. 16, no. 10, pp. 1005-1007, 2003.

[17] H. Schubert, "On the origin of 'anomalous' shapes of the separation curve in hydrocyclone separation of fine particles," Particulate Science and Technology, vol. 22, no. 3, pp. 219-234, 2004.

[18] J. G. Dueck, L. L. Min'Kov, and E. V. Pikushchak, "Modeling of the 'fish-hook' effect in a classifier," Journal of Engineering Physics and Thermophysics, vol. 80, no. 1, pp. 64-73, 2007.

[19] K. Nageswararao and R. A. Medronho, "Fish hook effect in centrifugal classifiers-a further analysis," International Journal of Mineral Processing, vol. 132, pp. 43-58, 2014.

[20] F. Bourgeois and A. K. Majumder, "Is the fish-hook effect in hydrocyclones a real phenomenon?" Powder Technology, vol. 237, pp. 367-375, 2013.

[21] J. Dück, L. Minkov, and T. Neesse, "A hydrodynamic model for enhanced sedimentation of small particles in a bidisperse suspension," Thermophysics and Aeromechanics, vol. 8, pp. 259269, 2001.

[22] J. Dueck, "The sedimentation velocity of a particle in a wide range of Reynolds numbers in the application to the analysis of the separation curve," Advanced Powder Technology, vol. 24, no. 1, pp. 150-153, 2013.

[23] L. L. Min'Kov and J. H. Dueck, "Numerical modeling of a nonmonotonic separation hydrocyclone curve," Journal of Engineering Physics and Thermophysics, vol. 85, no. 6, pp. 13171326, 2012.

[24] M. van Dyke, An Album of Fluid Motion, Stanford University, Stanford, Calif, USA, 1982.

[25] L. G. Loitsyanskii, Mechanics of Liquids and Gases, Pergamon Press, Oxford, UK, 1966.

[26] S. V. Patankar, Numerical Heat Transfer and Fluid Flow, Hemispheres, New York, NY, USA, 1980.

[27] L. Schiller and Z. Naumann, "A drag coefficient correlation," Zeitschrift des Vereines Deutscher Ingenieure, vol. 77, p. 318, 1935. 
[28] H. Schlichting, Grenzschicht-Theorie, Braun, Karlsruhe, Germany, 1965.

[29] S. Taneda, "Experimental investigation of the wake behind a sphere at low Reynolds numbers," Journal of the Physical Society of Japan, vol. 11, no. 10, pp. 1104-1108, 1956.

[30] C. Gerhart, J. Duck, and T. Neesse, "Fundamental study of the polydisperse suspensions in hydraulic classification," Mineral Processing, vol. 40, no. 7, pp. 328-334, 1999.

[31] S. Kumar, T. W. Pirog, and D. Ramkrishna, "A new method for estimating hindered creaming/settling velocity of particles in Polydisperse Systems," Chemical Engineering Science, vol. 55, no. 10, pp. 1893-1904, 2000. 


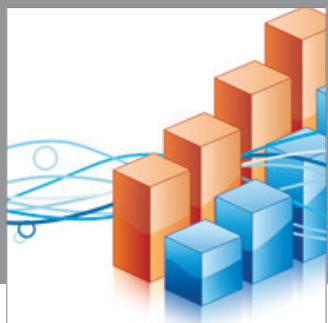

Advances in

Operations Research

mansans

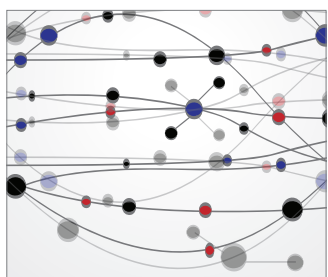

The Scientific World Journal
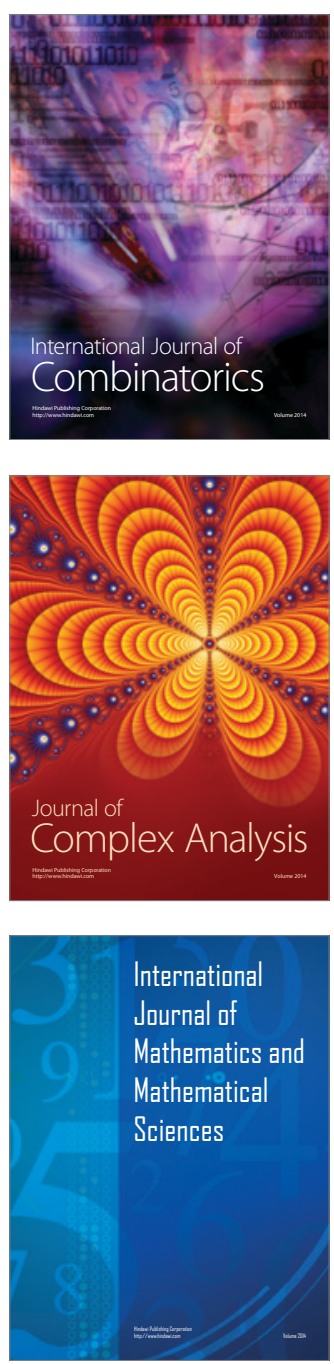
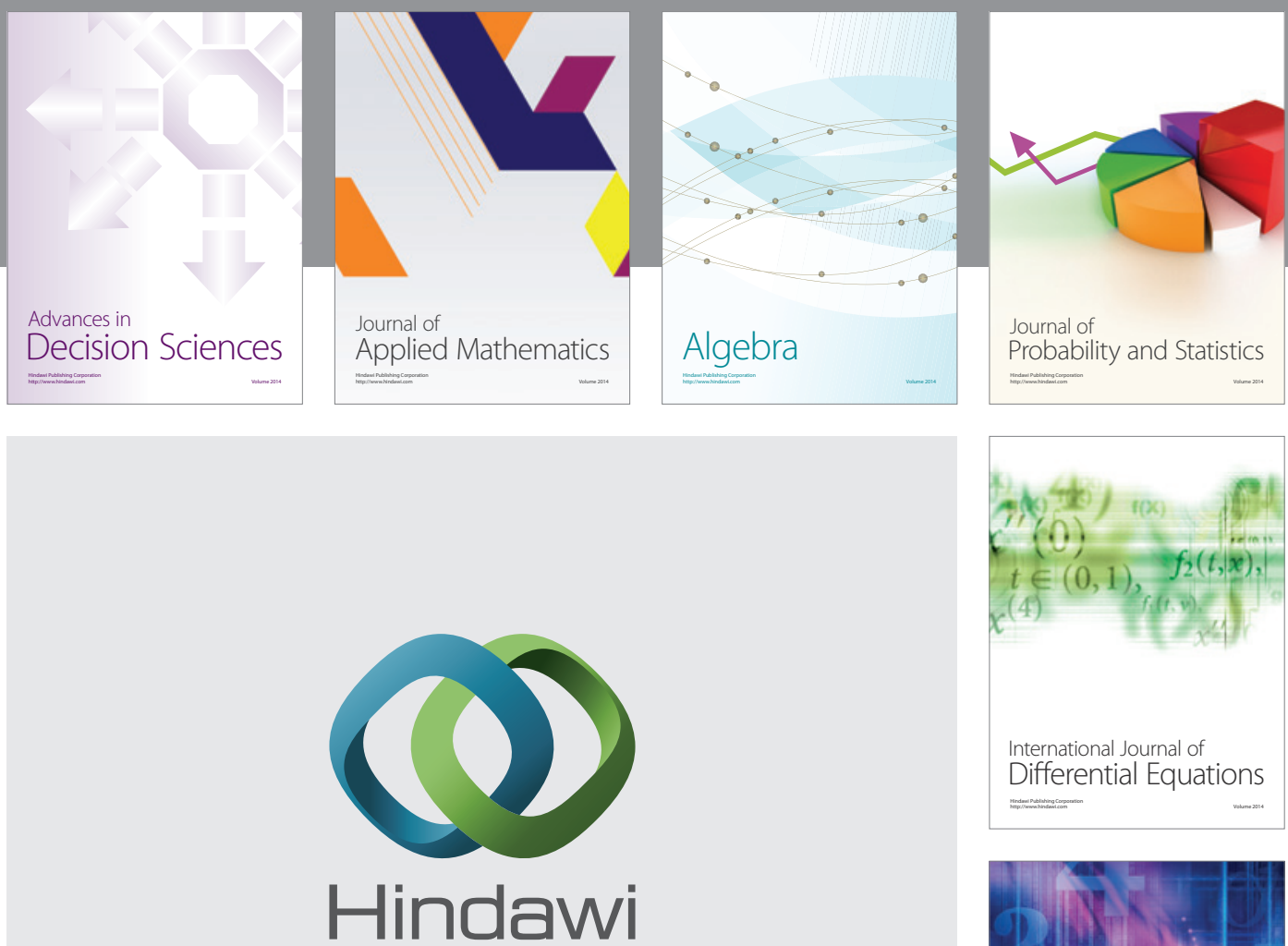

Submit your manuscripts at http://www.hindawi.com
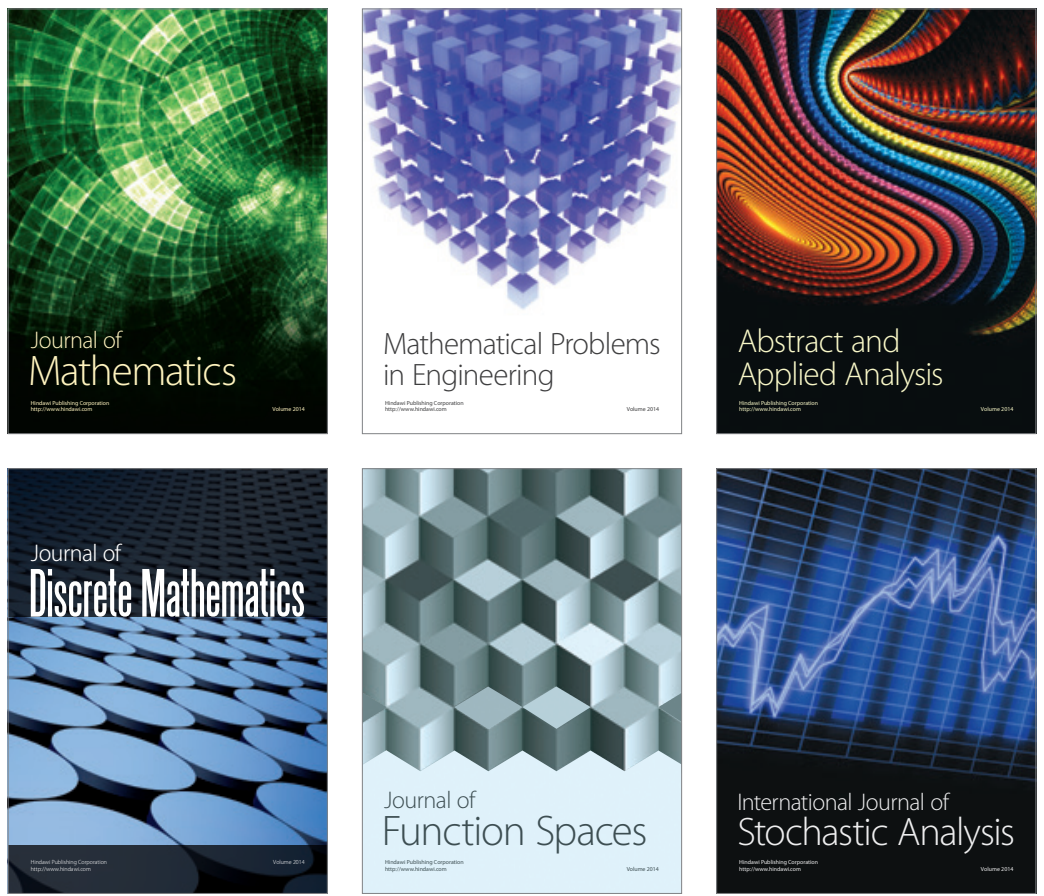

Journal of

Function Spaces

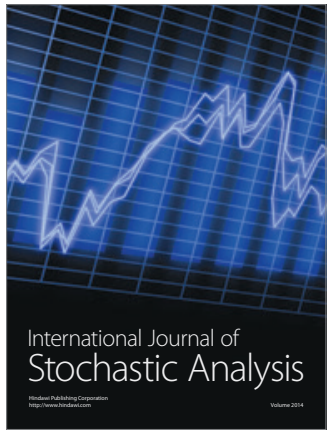

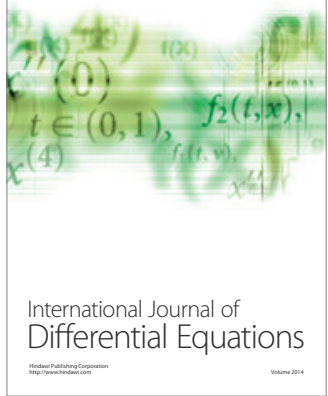
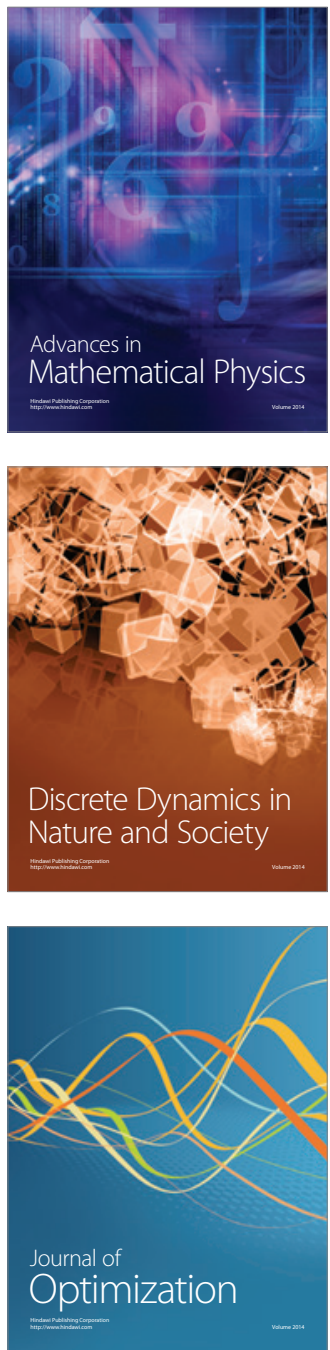